\title{
Low dose anti-thymocyte globulin versus basiliximab as induction in standard risk kidney transplant patients: 5-year follow-up
}

\author{
Candy Cahilog, Romina Danguilan, Paolo Miguel David, Mel-Hatra Arakama, Glenda Eleanor Pamugas
}

Department of Adult Nephrology, National Kidney and Transplant Institute, Quezon City, Philippines

Background: Kidney transplantation has significantly improved survival and quality of life of patients with end-stage renal disease and is considered the best form of renal replacement therapy. Rabbit anti-thymocyte globulin (rATG) has commonly been reserved for high immunologic risk patients and the resulting profound depression in immune response is associated with an increased risk for infection.

Methods: A retrospective cohort of patients transplanted from June 2012 to December 2014 was done to evaluate the short- and long-term outcome and efficacy of low dose rATG versus basiliximab as induction. CNI-based triple immunosuppression was given to the majority of patients.

Results: Among 165 patients in the study, 131 were given basiliximab and 34 were given low dose rATG (1-1.5 mg/kg/day for 3 days). The two groups were similar in mean age, native kidney disease, and cytomegalovirus (CMV) status. Higher proportion of patients who received rATG had positive panel reactive antibody $(P=0.0003)$, higher human leucocyte antigen mismatches $(P=0.0023)$, and non-related donors $(P=0.025)$. Low dose $r A T G$ and basiliximab as induction therapy resulted in comparable outcomes including DGF, graft function, proteinuria, incidence of acute rejection and post-transplant malignancy. Incidence of infection was significantly higher in basiliximab group at 3 -year post-KT: $11.5 \%$ vs. $0, \mathrm{P}=0.04$. CMV infection occurred very low at an overall rate of $0.6 \%$ at 1 - and 3 -year post-KT. Graft and patient survival at 5-year post-KT were $96.9 \%$ vs. $97.1 \%, P=0.99$ and $99 \%$ vs. $100 \%, P=0.57$, respectively.

Conclusions: Both low dose rATG and basiliximab induction resulted in excellent long-term outcomes. Low dose rATG was not associated with higher infection rates and can be used safely in standard risk patients.

Corresponding author: Candy Cahilog

E-mail: candycahilogmd@gmail.com

(C) The Korean Society for Transplantation

This is an Open Access article distributed under the terms of the Creative Commons Attribution Non-Commercial License (http://creativecommons.org/licenses/by-nc/4.0/) which permits unrestricted non-commercial use, distribution, and reproduction in any medium, provided the original work is properly cited. 